\title{
An Experimental Study on Transplantation of the Aortic Arch
}

Part I. Transplantation of the Aortic Arch under Severe Hypothermia (Rectal Temperature $18.5^{\circ}-14^{\circ} \mathrm{C}$ )

By

Takao Takahashi

(高橋 孝 雄)

From the Department of Surgery, Tohoku University, Faculty of Medicine, Sendai. Director: Prof. S-T. K atura

(Received for publication, April 22, 1957)

The author reported in the previous communication that under severe hypothermia, the heart could be successfully resuscitated after long cessation of the blood flow from the heart. In case the ascending aorta was divided and resutured, the technique was difficult. From these experimental results, considering less tension at the anastomosis and easy technique, transplantation of the aortic arch was carried out.

\section{EXPERIMENTAL}

\section{Method}

Anesthesia to obtain severe hypothermia

Same as that described in part I.

Cardiac resuscitation and warming

Same as that described in part I.

Operation

The chest was opened by a large incision encircling the scapula at the left 4 th intercostal space. The descending thoracic aorta was mobilized and the superior, occasionally the 2 nd intercostal artery was divided and ligated. The left brachiocephalic artery, then ductus Botalli was cut. The right brachiocephalic artery was freed, avoiding bilateral pneumothorax and finally the ascending aorta was dissected. Incising the pericardium longitudinally, the fat tissues at the reflecting portion of the pericardium were removed to completely free the aorta just above the aortic valve. A piece of tape was passed around the vagus and phrenic nerve to preserve them. Following interruption of the blood flow, clamps were placed on the right brachiocephalic artery and the descending thoracic aorta, and the aortic arch was removed en masse. With completion of anastomosis, the clamp on the ascending aorta was removed, 
and the heart was compressed several times. This procedure was done for the purpose to prevent air emboli, test the amount and degree of blood loss at the anastomosis. Postoperatively, rewarming and cardiac massage were both done at the same time. Prior to closure of chest, the pericardium was roughly sutured. Chest drainage tube was inserted before chest wall was sutured. Postoperatively, energetic chemotherapy was instituted.

Cessation of blood flow (Table I)

8 cases were used with interruption of the outflow tract, the aorta. In case interrupting the aorta, with consideration of its fragility, a Satinsky's clamp loosely covered with rubber was used.

A strong silk was tied on its tip to prevent mobilization of the clamp. One showed ventricular fibrillation during mobilization of the aortic arch.

Inflow tract and the aorta were both interrupted in 10 cases. The heart was rotated out intrapericardially and Nelaton's catheters were used to constrict the inferior vena cavae. Clamps were not used. The obstruction was released from the inflow tract in the order given.

Material for transplantation

$70 \%$ alcohol preserved homografts were used in 17 cases (Fig. 1.).

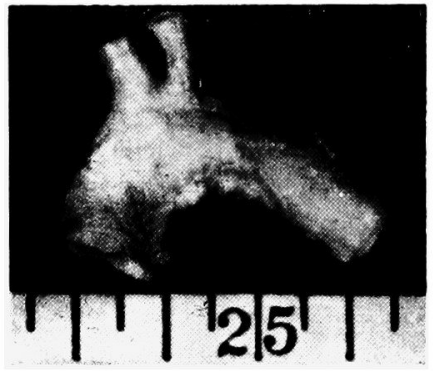

Fig. 1. Material used for transplantation. $(70 \%$ alcohol preserved homograft)

Aorta with same diameter was sclected. The left brachiocephalic artery was ligated at its origin beforehand. In one case, polyvinyl sponge was heated to make it poreless and made after an aortic arch exclusive of the left brachiocephalic artery with cuffs on both ends.

\section{Anastomosis}

Anastomosis was performed from the ascending aorta, the right brachiocephalic artery, and the descending thoracic aorta in the order given. First the anterior wall, then the posterior wall was sutured by a continous over and over stitches with 2 supporting sutures on both corners. Black silk No. 3 were used. 10\% sodium citrate was injected into the inside to prevent clot formation. The anastomosis of the ascending aorta 


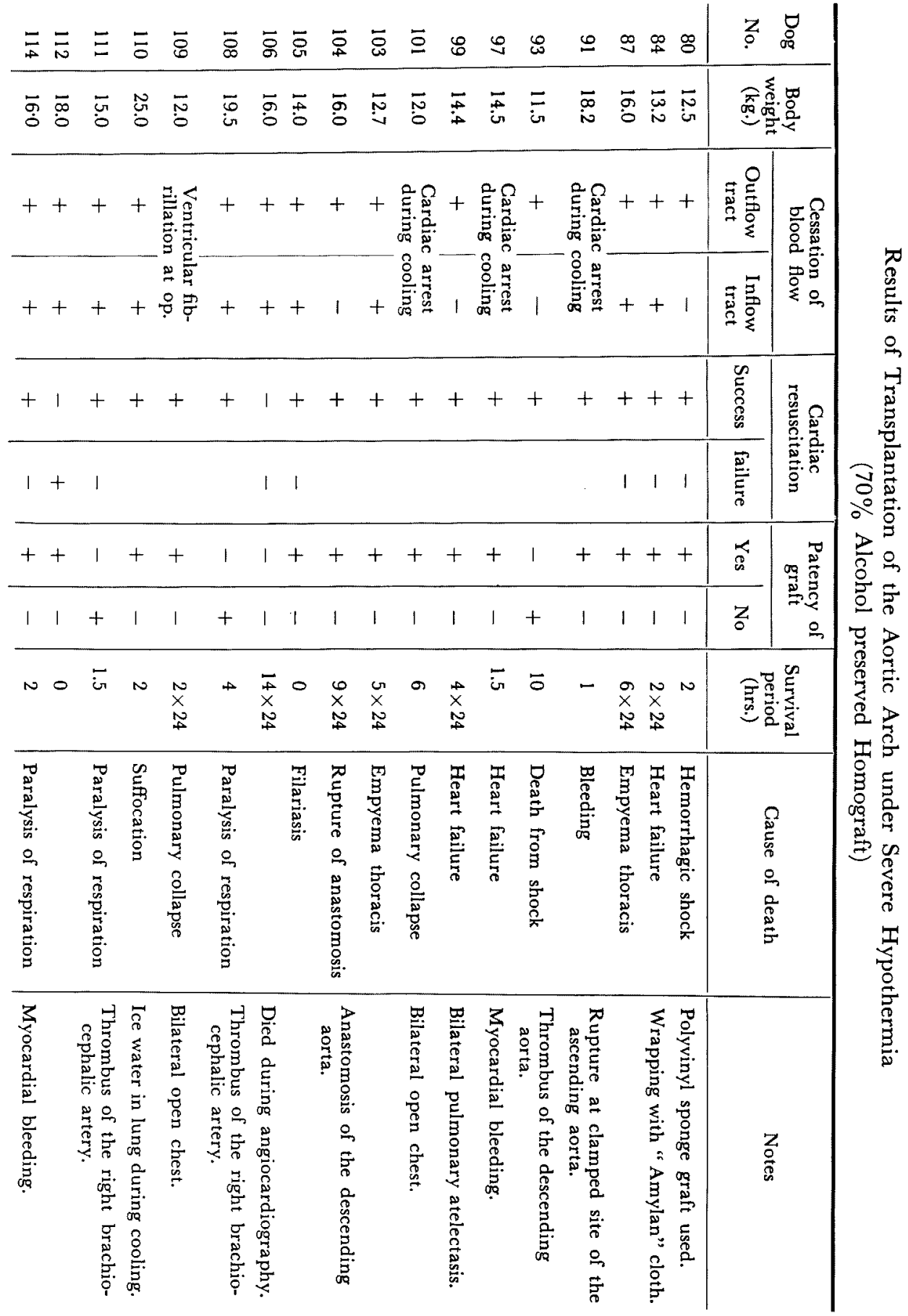


was made with rough interval (1.5-2 mm.), adapting the intima closely.

\section{Results}

5 of 18 dogs (Nos. 87, 99, 103, 104 and 106) survived 4-14 days, while the others died within 2 days. The cause of death is described in each case.

In spite of no blecding during cardiac massage after transplanting a graft made of polyvinyl sponge in No. 80, hemorrhage occurred from the entire graft after defibrillating the heart. In 84 "Amylan" was wrapped around the anastomosis of the ascending aorta to prevent bleeding. It died on the following night despite recovery of conciousness, movement of legs and reaction to pain stimuli. Dog Nos. 87 and 103 showed appetite on the 2nd day, walked on the 3rd day and reacted to petting. They died from empyema on the 5th and 6th postoperative day, respectively, despite energetic chemotherapy. Fig. 2 shows the re-

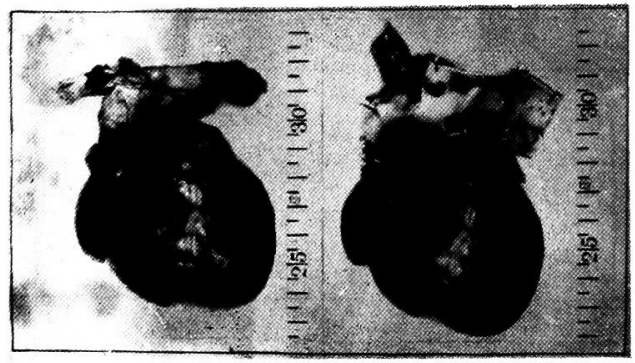

Fig. 2. Removed specimen in dog. No. 87.

moved specimen of No. 87. Operation was immediately started in No. 91 , because of cardiac arrest during cooling at rectal temperature $17^{\circ} \mathrm{C}$. Due to rough handling of the anastomosis of the ascending aorta, the clamped site was injuried, which caused fatal bleeding an hour later. Though No. 93 recovered from anesthesia, it died 10 hours later with spastic paraplegia of the hind legs. At autopsy, thrombus at the anastomosis obstructed the descending thoracic aorta. In Nos. 97 and 114, myocardial bleeding occurred during cardiac massage. Though it was successfully resuscitated, it died within a short time. No. 99 showed urinal and fecal excretion, started to walk on the 3rd day with no neurological disturbance, but died on the 4th day due to general weakness of the body. Nos. 101 and 109 both died 6 hours later and on the 2nd day, respectively. Both showed severe pulmonary collapse which was caused by bilateral pneumothorax during operation. Nos. 104 and 107 both showed a smooth postoperative course. However, the former died sud- 
denly by rupture at the descending thoracic aorta on the 9th day, while the latter succumbed to death on the 14th day during angiocardiography with rapid injection of Urocolin $15 \mathrm{cc}$. into the left ventricle under intravenous anesthesia with Ravonal. Good patency of the graft was recognized (Fig. 3). The entire course of EGG is reproduced in Fig. 4, which shows return to normal tracings on the 12th postoperative day. No. 105 showed normal heart beat only to become weak again, which didn't respond to injection of $2 \%$ calcium chloride. At autopsy, the ventricle and atrium were both filled with filaria. Nos. 108 and 111 died from respiratory arrest prior to cardiac standstill. Autopsy revealed thrombus obstructing the aorta at the anastmosis of the brachiocephalic artery.

Fig. 3

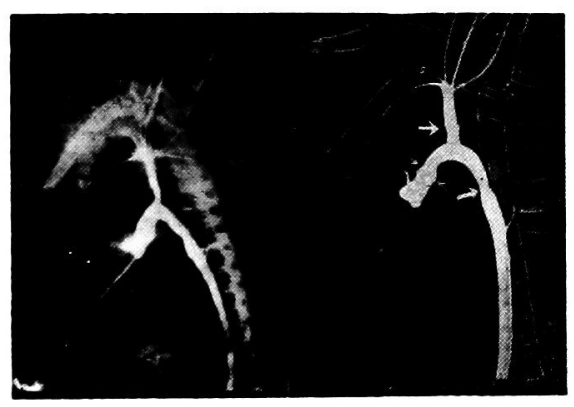

Fig. 4

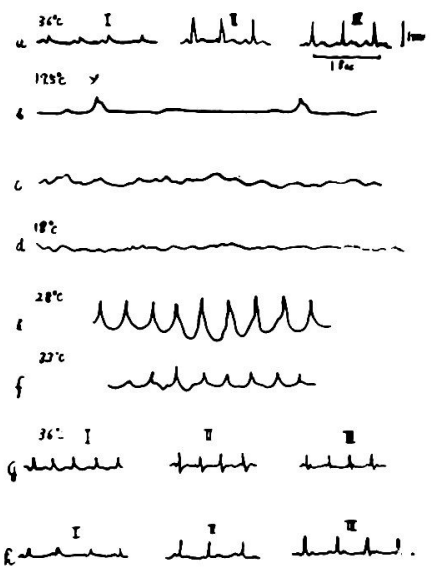

Fig. 3. Angiocardiography 14 days after transplantation of the aortic arch in dog No. 106. (sign $\uparrow$ in schema shows anastomosed part).

Fig. 4. Electrocardiographic tracing in dog No. 106.

Describing the patency at the anastomosis, 3 of 18 (Nos. 91, 111 and 108) showed obstruction of the lumen, of which 2 showed the smallest brachiocephalic arteries. In $\operatorname{dog}$ No. 108, the intima was injuried at the clamped site of the brachiocephalic artery (bulldog clamp was used in this case), from which extended a thrombus (Fig. 5). The remaining one showed thrombus at the anastomosis of the descending thoracic aorta. No obstruction was showed at the anastomosis of the ascending aorta. The remaining 15 were all patent.

Observing the relationship of cessation of cardiac circulation with the time for anastomosis, the shortest obstruction was 60 minutes, while the longest 140 minutes, average 88 minutes at rectal temperature $14^{\circ}-$ $18.5^{\circ} \mathrm{C}$. The time for anastmosis was 50 minutes the shortest, while 85 


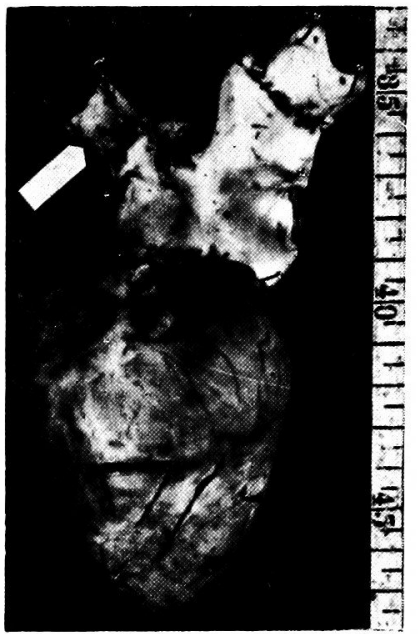

Fig. 5. Injury of intima at the clamped site of the right brachiocephalic artery $(\uparrow)$ in dog No. 108.

minutes the longest, average 60 minutes. The time to anastomose the ascending aorta required 14 to 47 minutes. The marked difference was due to resuture the frequent tear of the fragile aorta (Table II).

\section{TABLE II}

Relationship of Time for Anatomosis and Cessation of Blood Flow of Heart

\begin{tabular}{c|c|c|c}
\hline Dog No. & Rectal temp. $\left({ }^{\circ} \mathrm{C}\right)$ & $\begin{array}{r}\text { Time of interruption } \\
(\min )\end{array}$ & $\begin{array}{c}\text { Time for anastomosis } \\
\text { (min.) }\end{array}$ \\
\cline { 2 - 3 } 80 & 17.0 & 84 & 61 \\
84 & 18.0 & 82 & 70 \\
87 & 17.0 & 98 & 85 \\
91 & 16.5 & 105 & 62 \\
93 & 14.0 & 86 & 65 \\
97 & 14.5 & 140 & 50 \\
99 & 15.0 & 93 & 66 \\
101 & 16.0 & 100 & 58 \\
103 & 17.0 & 85 & 60 \\
104 & 18.5 & 65 & 52 \\
105 & 16.5 & 82 & 50 \\
106 & 17.5 & 60 & 52 \\
108 & 17.0 & 70 & 55 \\
109 & 17.0 & 65 & 54 \\
110 & 16.3 & 103 & 65 \\
11 & 16.0 & 65 & 50 \\
112 & 15.0 & 80 & 65 \\
114 & 18.0 & 105 & 50 \\
& & &
\end{tabular}

In 1955, Cooley et al. ${ }^{11)}$ reconstructed the blood vessel after resecting 
an aneurysm of the ascending aorta with aid of a shunt. They pointed out the significance of blood to the brain and rapid increase of burden on heart following interruption of the ascending aorta. The following points were suggested to solve this problem.

1. In order to divide the aortic arch, shunts should be used to diminish increase of load on the heart, and prevent interruption of blood flow to the brain and peripheral organs, or the interruption should be limited as short as possible.

2. With use of hypothermia, both decrease of cardiac output and tissue oxygen consumption are obtained.

When shunt is used to prevent overload on the heart, tubes with various diameters are used. Izant et al. ${ }^{2)}$ demonstrated that in dogs shunt having inner diameter $5 \mathrm{~mm}$. will permit $80 \%$ of blood flow to the periphery without inducing anoxia to the distal vital organs. Stranahan et $a .^{3)}$ reported without definite conclusions that $10 \mathrm{~mm}$. would be the minimum diameter required for shunts in clinical cases. In a few cases, the author used shunts under hypothermia as Satinsky reported. ${ }^{4}$ We used the back flow from the left ventricle caused by artificial mitral insufficiency, but all failed except those with inner diameter 6-8 mm. When this method is used, the technique is complicating, shunts and clamps will obstruct the operative field, danger of massive bleeding is imminent, and fine technique is required, due to limit of cessation of blood flow. According to Satinsky, ") the ascending aorta must be anastomosed within 11-20 minutes. As the ascending aorta is fragile and heart beat is strong, the danger to injure the blood vessel by overload on the heart can't be denied. According to Alley et al., ${ }^{5}$ ) completion of procedure was obtained in 9 out of 20 , of which 5 of 11 were lost when shunt was sutured to the ascending aorta.

On the other hand, hypothermia has been applied in vascular surgery. Reports to apply hypothermia to prolong the cessation of the thoracic aorta have been published.6) However, due to frequent occurrence of ventiricular fibrillation, severe hypothermia below $25^{\circ} \mathrm{C}$ seems to be discarded for use. Watanabe et al. ${ }^{7}$ at this clinic have been studying severe hypothermia for 2 years, who have reported that ventricular fibrillation is not a serious problem under severe hypothermia, if appropriate method for resuscitation is at hand. They have been successful in an epoch making experiment to resuscitate a dog which had interruption of blood flow to the brain for 2 hours and 40 minutes, without heart beat and respiration. No serious postoperative sequelae was found on ECG, EEG, histology (heart, brain and other vital organs) and neurology. Though it is repeated again, applying severe hypothermia, the ajkthor devised a new method to transplant the aortic arch directly under long 
cessation of cardiac circulation with arrested heart action, which is an entirely different method using various shunts by others. Hardin ${ }^{81}$ lost 9 of 11 within a short time, of which 7 were in cardiac failure with arrhythmia, heart dilatation and ventricular fibrillation during operation. In case shunts are used, it seems to be difficult to restore normal rhythm when ventricular fibrillation occurrs, and to maintain effective blood pressure, which are both disadvantages of using shunts.

As described before, the time for anastomosis is 60 minutes, while that of obstruction of the heart flow 88 minutes. This difference shows that anastomosis can be done with leisure. In case of clinical application, because of unavoidable prolongation of time for anastomosis due to the anatomical difference in dog from human beings, it is the greatest advantage of using severe hypothermia.

Severe hypothermia is studied by Gollan $e t$ al. ${ }^{91}$ and Schaefer et $a l .^{10)}$ in the U.S.A. and Watanabe et al. ${ }^{71}$ in Japan. It is, however, not yet applied in clinical cases, but its clinical application is within near future. The possibility of applying transplantation of aortic arch which author experimented on clinical cases is demonstrated. This method will contribute to the advance of vascular surgery together with the conventional method using shunts.

\section{SUMMARY}

The author transplanted the aortic arch with homologus grafts under long cessation of the blood flow from the heart as one of the applications of severe hypothermia which is presently studied by Watanabe $e$ t al. at this clinic. The blood flow of the heart was interrupted for a long time, $60-140$ minutes, at rectal temperature $18.5^{\circ}-14^{\circ} \mathrm{C}$. Five of 18 survived the operation with successful outcome. Anastomosis could be carried out without tension and fear of massive blood loss, and it had the advantage to perform careful operation.

\section{References}

1) Cooley, Mahaffey \& DeBakey, Surg. Gynec. \& Obst., 1955, 101, 667.

2) Izant, Hubay \& Holden, Surg., 1953, 33, 233,

3) Stranahan, Alley, Sewell \& Kausel, J. Thorac. Surg., 1955, $29,54$.

4) Satinsky, Neptune \& Alai, Ann. Surg., 1955, 141, 38.

5) Alley, Sewell, Formel, Stranahan, Kausel \& Koth, Surgical forum, W. B. Sauders Co., Phil., 1954, p. 85.

6) Takahashi, Ohara \& Wada, Jap. J. Thorac. Surg. (Jap.), 1956, 9, 732.

7) Watanabe, Okamura, Takahashi \& Noro, Sogo Igaku (Jap.), 1956, 31, 771.

8) Hardin, Batchelder \& Schaefer, Surg., 1952, 32, 219.

9) Gollan, Tysinger, Grace, Kong \& Meneely, Am. J. Physiology, 1955, 181, 297.

10) Schaefer, Hughes \& Barila, A.M.A. Arch. Surg., 1955, 70, 723. 